\title{
Mesenchymal Stem Cells Provide Neuroprotection by Regulating Heat Stroke-Induced Brain Inflammation
}

\author{
Yu Zhang ${ }^{1}$, Zihui Deng ${ }^{2+}$, Yun $\mathrm{Li}^{1+}$, Rui Yuan ${ }^{1}$, Mengmeng Yang ${ }^{1}$, Yan Zhao ${ }^{1}$, Lu Wang ${ }^{1}$, \\ Feihu Zhou ${ }^{1}$ and Hongjun Kang ${ }^{1 *}$ \\ ${ }^{1}$ Department of Critical Care Medicine, General Hospital of the Chinese People's Liberation Army, Beijing, China, \\ ${ }^{2}$ Biochemistry Department of Graduate School, General Hospital of the Chinese People's Liberation Army, Beijing, China
}

\section{OPEN ACCESS}

Edited by:

George E. Barreto,

University of Limerick, Ireland

Reviewed by:

Johannes Boltze,

University of Warwick,

United Kingdom

Eun Hee Kim

Sungkyunkwan University,

South Korea

${ }^{*}$ Correspondence:

Hongjun Kang

doctorkang301@163.com

tThese authors have contributed equally to this work

Specialty section:

This article was submitted to Neurotrauma,

a section of the journal

Frontiers in Neurology

Received: 04 February 2020

Accepted: 14 April 2020

Published: 05 May 2020

Citation:

Zhang Y, Deng Z, Li Y, Yuan $R$ Yang $M$, Zhao $Y$, Wang L, Zhou F and Kang H (2020) Mesenchymal Stem

Cells Provide Neuroprotection by Regulating Heat Stroke-Induced Brain Inflammation. Front. Neurol. 11:372.

doi: 10.3389/fneur.2020.00372
Heat stroke $(H S)$ is the most acute type of heat illness accompanied with serious central nervous system (CNS) dysfunction. Despite the pathological process being clearly studied, effective treatment is deficient. Currently, mesenchymal stem cells (MSCs) have been demonstrated to have neuroprotective effects as there are no old ones. Thus, the purpose of the present study was to explore the neuroprotective effects and mechanisms of MSCs against HS-induced CNS injury. HS in rat models was induced by a high-temperature environment and treated with MSCs via the tail vein. The results demonstrated that MSC injection significantly reduced the mortality and inhibited the circulation inflammatory response. Moreover, the HS-induced neurological deficit and neuronic damage of the hippocampus were significantly ameliorated by MSC administration. In addition, MSC administration significantly restored astrocytes and inhibited cerebral inflammatory response. These results indicate that MSC infusion has therapeutic effects in HS of rats by regulating the circulation and cerebral inflammatory response. Moreover, astrocytes increased in MSC-treated HS rats when compared with the untreated ones. This may suggest a potential mechanism for HS prevention and therapy through MSC administration.

Keywords: heat stroke, mesenchymal stem cell, neuroinflammation, astrocyte, inflammatory reactions

\section{INTRODUCTION}

Heat stroke (HS) is the most serious type of heat illness which refers to the presence of hyperthermia (core body temperature that rises above $40^{\circ} \mathrm{C}$ ) and central nervous system (CNS) dysfunction $(1,2)$. The morbidity of HS increases yearly and its mortality is over $60 \%(3$, 4). In the early stages of HS, all patients have CNS injury, while more than $30 \%$ will then progress to long-term CNS dysfunction as sequela $(1,3,5)$. The cerebellum and hippocampus of the brain are particularly vulnerable to HS which would induce a high rate of mortality or permanent neurological sequelae. Moreover, previous studies have identified that HS is a form of hyperthermia associated with a systemic inflammatory response that results in a syndrome of multi-organ dysfunction, coagulation activation, and fibrin formation which clinically manifests as disseminated intravascular coagulation (DIC) $(1,6,7)$. In the CNS, HS induces hypotension and intracranial hypertension which reduces blood flow to the brain and results in cerebral ischemia, hypoxia, and neuronal damage. This neurotoxic cascade involves an overproduction of reactive oxygen species (ROS), cytokines, glutamate, and calcium ion $(7,8)$. Another study indicated that HS can decrease learning ability and memory in rats, the mechanisms of which 
may be related to changes of iron levels in the hippocampus (9). Despite pathogenesis and treatment having been deeply studied, including rapid, and effective cooling followed by close monitoring, blood purification therapy for renal/hepatic failure, continuous electroencephalogram (cEEG) monitoring, and the use of anticoagulants, unfortunately, no effective and comprehensive management for HS-induced organ dysfunction, especially for the CNS, has been developed $(3,10)$.

Currently, mesenchymal stem cell (MSC)-based therapy is being considered as a potential treatment strategy for CNS injury (11-13). MSCs possess multipotent properties, such as the capacity to secrete various factors, differentiation potential, and immunoregulatory effects (14). Previously, numerous studies on CNS injuries in animal models have shown that infused MSCs can ameliorate brain injuries (11-13). MSCs can protect against cerebral ischemic injury by preventing neuronal damage via the inhibition of apoptosis (15). Other animal experiments have demonstrated that MSCs play a potential neuronal protective effect in stroke, traumatic brain injury, multiple sclerosis, nerve injury, and neurodegenerative diseases via the secretion of neurotrophic factors, regulation of inflammatory reaction, modulation of ion metabolism, and so on $(12,13,16,17)$. These properties of MSCs make them excellent candidates for CNS injury management. Although previous studies have shown that mesenchymal stem cells could improve survival following HS in animals $(12,18,19)$, the protective effects of brain injuries after HS has not been clearly demonstrated.

Therefore, in order to validate the effects of MSCs on brain injuries during HS, the present experiment was performed to assess the effects on survival, metabolic functions, neurons and astrocytes, and the plasma and brain levels of cytokines in rats with or without MSC administration after HS injury.

\section{MATERIALS AND METHODS}

\section{Animals}

A total of 90 adult (aged 8 weeks) and 20 immature (aged 4 weeks) male Sprague-Dawley (SD) rats were supplied by the Experimental Animal Center of the General Hospital of PLA. All animal experiments were approved by the Animal Care and Use Committee of the General Hospital of PLA and were conducted in accordance with the NIH Guide for the Care and Use of Laboratory Animals. Animals were maintained in a room with a 12/12-h light/dark cycle and an ambient temperature of $22-25^{\circ} \mathrm{C}$. All animals had free access to food and water. At the end of the experiment, the rats were anesthetized with pentobarbital sodium intraperitoneally $(60 \mathrm{mg} / \mathrm{kg})$ and sacrificed by cervical dislocation.

\section{Adipose-Derived MSC Isolation, Culture, and Identification}

Adipose-derived MSCs were isolated and purified from the immature rats as previously described (20). Briefly, the rats were anesthetized with pentobarbital sodium intraperitoneally $(60 \mathrm{mg} / \mathrm{kg})$ and sacrificed by cervical dislocation. The adipose tissue isolated from the groin was cut into pieces and digested using $0.05 \%$ trypsin and $0.1 \%$ collagen $\mathrm{I}$. The digest solution was filtered and centrifuged. After that, cells were washed twice and cultured in a low glucose DMEM (Gibco, Gran Island, NY, USA) supplemented with $10 \%$ fetal bovine serum (Gibco, Gran Island, NY, USA), penicillin (80 units/ml), and streptomycin $(0.2 \mathrm{mg} / \mathrm{ml})$. MSCs were identified as previously described (20). Immunologic phenotypes of third passage (P3) MSCs were analyzed by flow cytometry. Once an $\sim 80 \%$ confluence was reached, cells were collected and counted, then were randomly divided into six groups (one group per antibody), each containing $1 \times 10^{6}$ cells. Cells were then washed with PBS and incubated in the dark for $15 \mathrm{~min}$ at room temperature with the following antibodies: Allophycocyaninconjugated CD90 (1:20; cat. no. 561409; BD Biosciences, San Jose, CA, USA), R-phycoerythrin-conjugated CD54 (1:20; cat. no. 554970; BD Biosciences), fluorescein isothiocyanate (FITC)conjugated CD44 (1:20; cat. no. 550974; BD Biosciences), FITCconjugated CD34 (1:20; cat. no. 11-0341-82; eBioscience; Thermo Fisher Scientific, Inc.), FITC-conjugated CD11b (1:20; cat. no. 554982; BD Biosciences), and FITC-conjugated CD45 (1:20; cat. no. 554877; BD Biosciences). Following incubation, cells were washed with PBS and then analyzed by flow cytometry, which was performed using a BD Accuri C6 software system (version 1.0.264.21; BD Biosciences).

In order to perform differentiation potential analysis, P3 MSCs were cultured in a six-well plate at a density of $10^{4}$ cells/well at $37 \mathrm{C}$ in an atmosphere of $5 \% \mathrm{CO}_{2}$ and relative humidity of $\sim 100 \%$. Once a $\sim 70$ or $\sim 100 \%$ confluency for osteogenic or adipogenic differentiation was reached respectively, the medium was replaced with SD rat MSCs adipogenic (cat. no. RASMD-90031) or osteogenic (cat. no. RASMD-90021) differentiation medium (Cyagen Biosciences Inc., Guangzhou, China). Then at $37 \mathrm{C}$ in an atmosphere of $5 \% \mathrm{CO} 2$ and relative humidity of $\sim 100 \%$, the cells were cultured for 2 weeks. Following a fixation using $4 \%$ paraformaldehyde at room temperature for $30 \mathrm{~min}$, adipogenic differentiation was detected by staining with $0.5 \%$ Oil red O (Sigma-Aldrich; Merck $\mathrm{KGaA}$, Darmstadt, Germany) at room temperature for $1 \mathrm{~h}$, and osteogenic differentiation was detected via staining with $0.1 \%$ alizarin red $\mathrm{S}$ ( $\mathrm{pH}$ 4.2; Sigma-Aldrich; Merck KGaA) at room temperature for $30 \mathrm{~min}$. An inverted microscope was used to observe the cells. The identification results of the cells are displayed in Supplementary Figure 1. The P2 MSCs cells were cryopreserved, and before intravenous infusion, they were resuscitated and then recultured into P4. Freshly harvested, the early passage (P4) MSCs were used in all subsequent experiments.

\section{HS Injury Rat Models Induction and Treatment}

A HS injury model was established as previously described (21). Briefly, the rats (without anesthesia) were maintained in a $40^{\circ} \mathrm{C}$ and $80 \%$ humidity environment. The rectal temperature of rat was monitored continuously. When temperatures reached $42^{\circ} \mathrm{C}$ (about $60 \mathrm{~min}$ ), the rat was returned to room temperature and an intraperitoneal injection of $5 \mathrm{ml}$ physiological saline was 


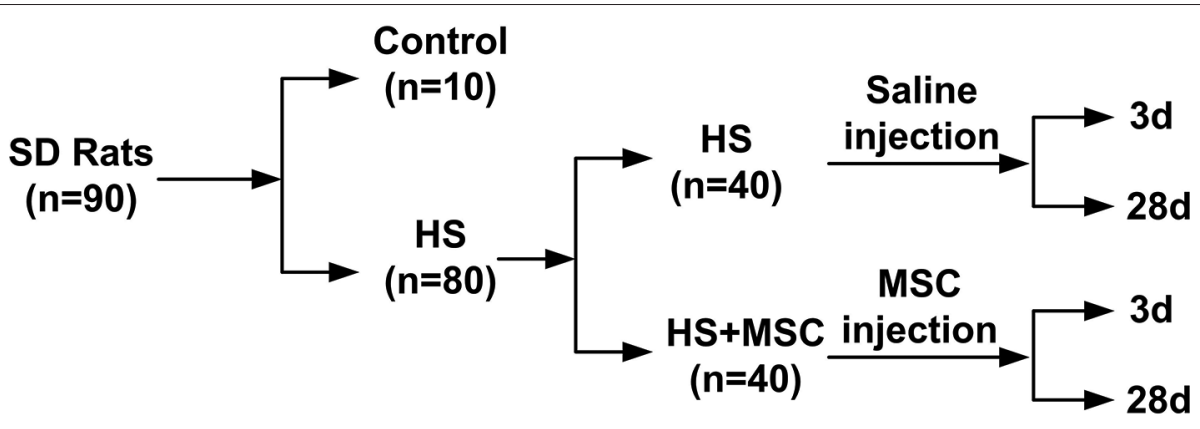

FIGURE 1 | Study design.

administered. Rats with paralysis and unconsciousness were identified as qualified HS injury models. The verified HS injury model rats were randomly divided into an HS group and an MSC-treated group ( $n=40$ in each group). Each rat in the treated group was infused with $2 \times 10^{6}$ MSCs suspended in $0.3 \mathrm{ml}$ physiological saline via the tail vein. The rats in the HS or MSC-treated groups were further randomly divided into early stage (3d) and late stage (28d) ( $n=20$ in each group). Besides, before HS injury models were built, 10 rats were randomly selected as control group and they were maintained at room temperature and only infused with $0.3 \mathrm{ml}$ physiological saline (Figure 1). The rats were observed for 28 days after HS injury, with or without MSC infusion, to estimate the survival rates.

\section{Neurological Deficit Evaluation}

Each rat in the different groups was evaluated for neurological deficits according to a modified Neurological Severity Score system containing motor (limb shape and walking posture), sensory (visual, tactile, and proprioceptive), reflex, and balance tests (22). Neurological function was graded on a scale of $0-$ 10 , and higher scores represent heavier injuries. Neurological Severity Score was assessed at the designated time point (i.e., 3 and $28 \mathrm{~d}$ ).

\section{Determination of the Effects of Infused MSCs on Cytokines in HS Injury Rats}

For determination of interleukin-1 $\beta$ (IL-1 $\beta$ ), interleukin6 (IL-6), interleukin-10 (IL-10), tumor necrosis factor $\alpha$ (TNF- $\alpha$ ), monocyte chemoattractant protein 1 (MCP-1), and growth-related oncogene (GRO), Rantes in the blood or brain tissues were taken at 3 days (early phase) and 28 days (late phase) after MSC infusion. Blood samples were centrifuged at $2000 \mathrm{~g}$, for $10 \mathrm{~min}$, at $4^{\circ} \mathrm{C}$, and the supernatants were harvested. The brain samples were homogenized in 10 volumes of ice-cold PBS. The homogenates were centrifuged at $12,000 \mathrm{~g}$, for $15 \mathrm{~min}$, at $4^{\circ} \mathrm{C}$. The supernatants were stored at $-80^{\circ} \mathrm{C}$ until measurement. The concentration of IL- $1 \beta$, IL-6, IL-10, TNF- $\alpha$, MCP-1, and GRO, Rantes in the blood and tissue lysates were determined using Procarta $\mathrm{Plex}^{\mathrm{TM}}$ Analyst 1.0 (eBioscience, San Diego, USA) according to the manufacturers instruction.

\section{Histological Examinations}

Perfusion fixation was performed to prepare brain tissue specimens at different time points after MSC infusion. Briefly, the rats were anesthetized and prepared with PBS or $4 \%$ paraformaldehyde. The brain tissues were dissected and immersed in $4 \%$ paraformaldehyde for $12 \mathrm{~h}$ and then immersed in a $30 \%$ of sucrose solution for $24 \mathrm{~h}$ at room temperature. The brain tissues were then frozen in a cooled embedding medium (OCT, Sakura Finetek USA, Inc.) and sliced into $7 \mu \mathrm{m}$-thick sections using a freezing microtome (Leica Microsystems, Wetzlar, Germany) for staining. The sections were stained with hematoxylin and eosin (HE) or Nissl's staining. These stained sections were visualized and scanned with a Panoramic MIDI CaseViewer System (3DHISTECH, Hungary).

\section{Immunofluorescence Staining}

For immunofluorescence staining, polyclonal anti-glial fibrillary acidic protein (anti-GFAP) (1:100, Santa Cruz, CA, USA) was used as the primary antibody to identify GFAP expression or astrocytes. Briefly, sections were fixed in a cooled acetone for $15 \mathrm{~min}$ and washed with PBS twice. The sections were incubated with a $1 \times$ blocking buffer (Cell Signaling Technology, Inc., Danvers, MA, USA) at $37^{\circ} \mathrm{C}$ for $1 \mathrm{~h}$, and then incubated with a primary antibody overnight at $4^{\circ} \mathrm{C}$, followed by further incubation for $1 \mathrm{~h}$ at $37^{\circ} \mathrm{C}$ with IgG Fab2 Alexa Fluor (R) 555 immunoconjugated secondary antibody at a 1:1000 dilution (Cell Signaling Technology, Danvers, MA, USA). The nuclei were stained with 4', 6-diamidino-2-phenylindole (DAPI, Vector Laboratories, Burlingame, CA, USA) for $15 \mathrm{~min}$ at room temperature. Negative controls were processed simultaneously by replacing the antibodies with PBS. The sections were visualized and scanned with a Panoramic MIDI CaseViewer System (3DHISTECH, Hungary).

\section{Statistical Analysis}

SPSS 19.0 software was used to process the data. All experiments (biological replicates) were performed at least three times independently. Data were expressed as mean \pm standard deviation. One-way ANOVA with Tukey's post-hoc analysis was used when data passed the test for 

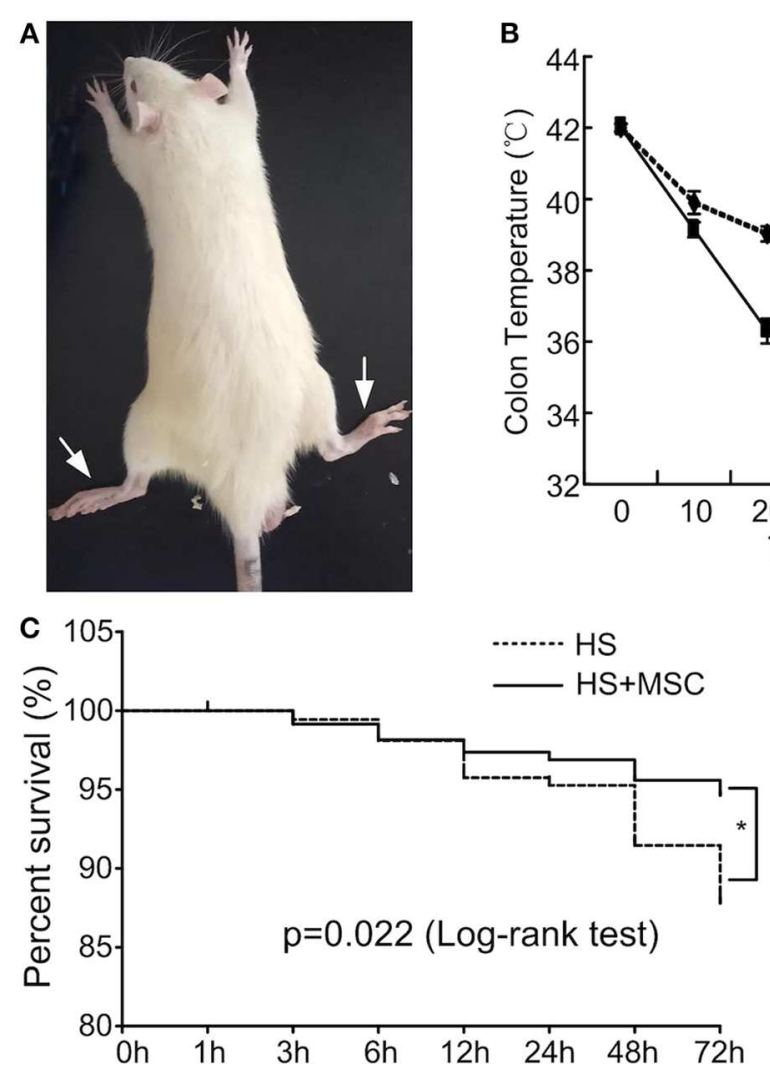
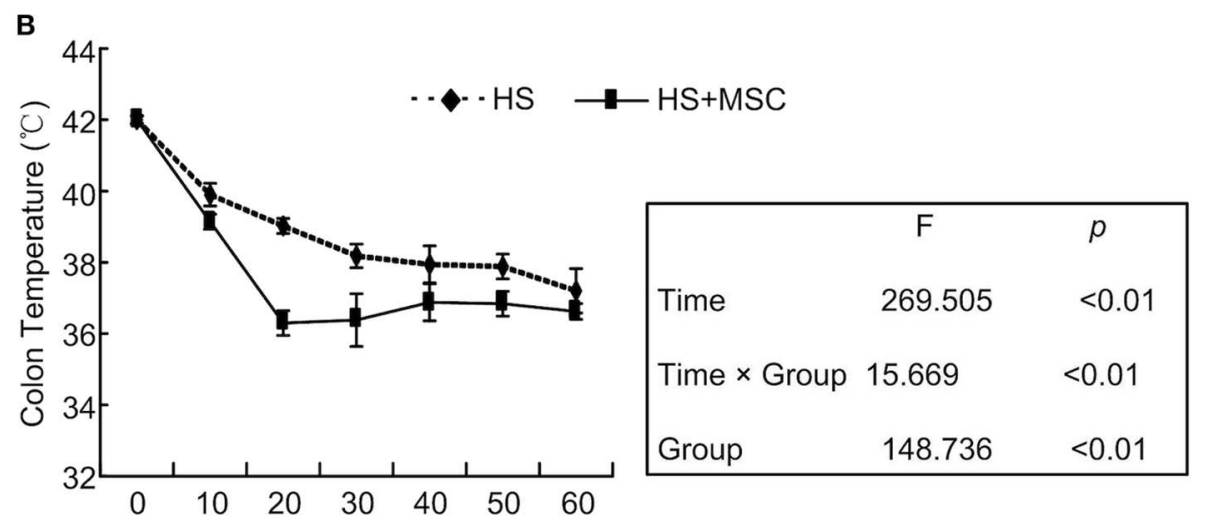

Time (min)

FIGURE 2 | MSC infusion ameliorated the survival rate of HS rats. When the rectal temperature reached $42^{\circ} \mathrm{C}$, the rat was returned to room temperature and an intraperitoneal injection of $5 \mathrm{ml}$ physiological saline was administered. The HS rats were observed and validated to have acute hindlimb paralysis (A). After MSCs administration, the rectal temperature of all HS rats was monitored continuously (B). Moreover, the survival rate in the early (3 days) (C) and long-lasting stages (28 days) (D) was assessed. $n=40$ in each group. ${ }^{*} P<0.05$ vs. HS group.

normality and equal variance. If not, non-parametric statistical analysis (Wilcoxon signed-rank test) was used. Data for colonic temperature variables were analyzed using a repeated measures analysis of variance (timexgroup). A $p$-value of $<0.05$ was considered to indicate a statistically significant difference.

\section{RESULTS}

\section{MSC Infusion Increased the Survival Rate of HS Rats}

The HS rats were observed and validated to have acute hindlimb paralysis (Figure 2A). Following MSC administration in the HS rats, colonic temperature decreased quickly when compared with the HS group rats (Figure 2B). Moreover, in the different stage phases, untreated HS rats demonstrated a higher mortality, whereas following the infusion of MSCs, the survival rate demonstrated a significant amelioration both in the early (3 days, Figure 2C) and long-lasting stages (28 days, Figure 2D). These results indicate that HS injury induced a paralysis symptom and high mortality in the rats, which could be ameliorated by MSC infusion.

\section{MSC Administration Modulated Pro-inflammatory and Anti-inflammatory Reactions of HS Rats}

As shown in Figure 3, following HS injury, the serum IL-10 levels were significantly decreased but IL- $1 \beta$, IL- 6 , and TNF- $\alpha$ levels were significantly increased at 3 days as compared with the control rats $(P<0.01)$. This lasted until 28 days, where the IL-10 levels were still notably lower than that of control group $(P<0.01)$. However, they were significantly restored compared with the rats of the 3 -day group $(P<0.01$, Figure 3A). The IL-1 $\beta$ levels were approaching those of the control group and significantly decreased at 28 days when compared with the rats of 3-day group ( $P<0.01$, Figure 3B). The IL-6 and TNF- $\alpha$ levels were still notably higher than those of the control group $(P<0.01)$, but both significantly decreased compared with rats of the 3-day group $(P<0.01$, Figures 3C,D). MSC-treated HS rats demonstrated a significant improvement in their levels of serum inflammatory cytokine. Compared with untreated rats, the serum IL-10 levels of the MSC-treated rats were significantly increased at 3 days and had no significant differences at 28 days (Figure 3A). The IL-1 $\beta$, IL-6, and TNF- $\alpha$ levels of the MSCtreated rats were all significantly decreased when compared with those of the untreated rats at 3 days but showed no differences 

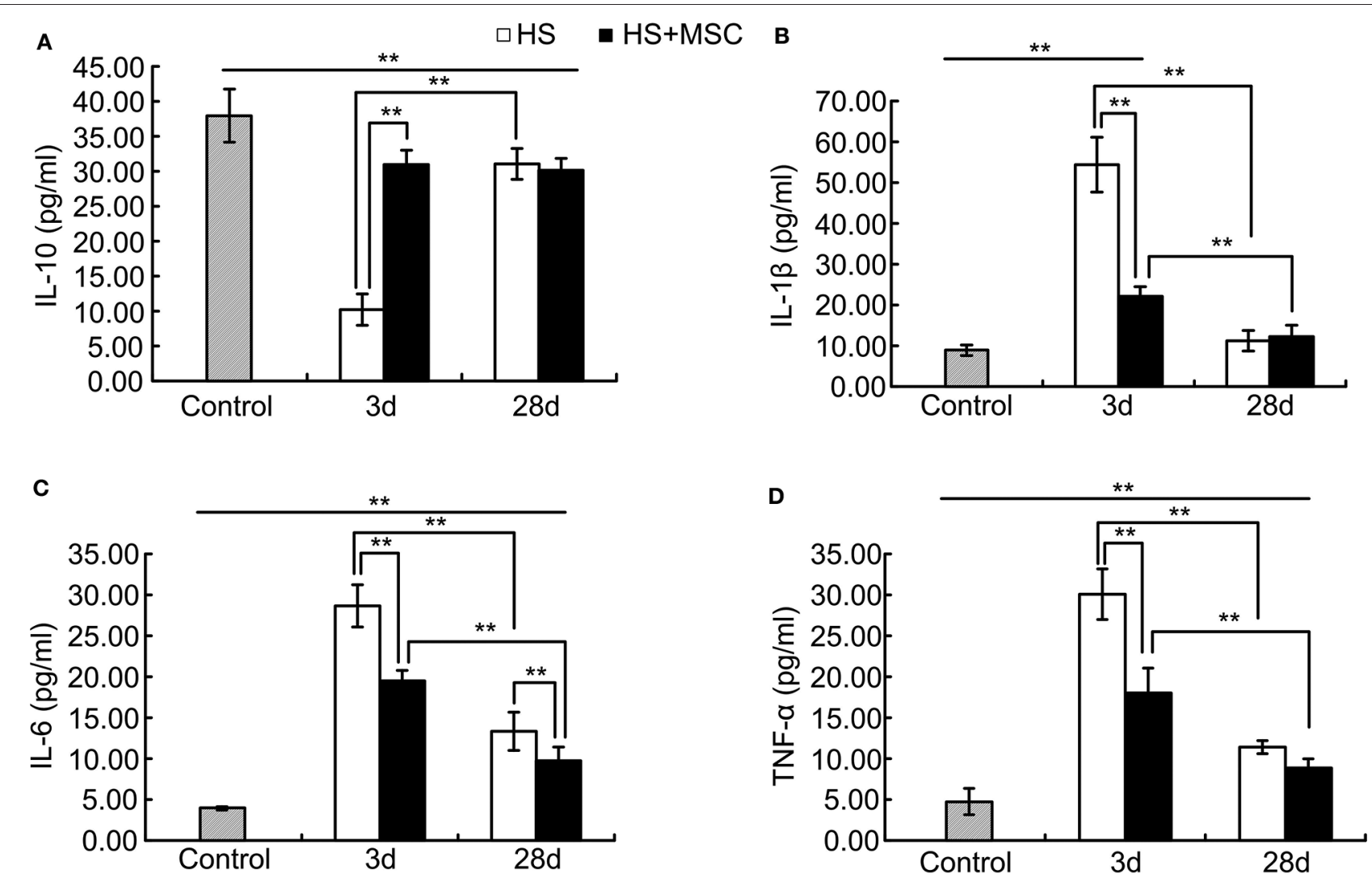

FIGURE 3 | MSCs modulated pro-inflammatory and anti-inflammatory reactions of HS rats. At different time points after MSCs or saline infusion, the blood of rats in each group was collected, and the IL-10 (A), IL-1 $\beta$ (B), IL-6 (C), and TNF- $\alpha$ (D) levels in the rat's blood serum were assayed. $n=10$ rats per group. ${ }^{\star \star} P<0.01$.

at 28 days except in IL-6 (Figures 3B-D). The IL-6 levels of the MSC-treated rats were still significantly decreased when compared with those of the untreated rats at 28 days (Figure $3 \mathrm{C}$ ). These data demonstrate that MSC administration can modulate pro-inflammatory and anti-inflammatory reactions of HS rats.

\section{MSC Administration Provided Neuroprotective Effects for HS Rats}

HS rats exhibited significantly increased neurological deficit scores at both 3 days and 28 days when compared with those of the control rats (both $P<0.01$ ) (Figure 4A). However, the scores were significantly decreased at 28 days as compared with rats of the 3 -day group $(P<0.01)$. MSC-treated HS rats also exhibited increased neurological deficit scores at both 3 and 28 days when compared with the control rats $(P<0.01)$, and the scores were notably decreased when compared with the same time point for untreated HS rats (both $P<0.01$ ). Moreover, the hippocampal histopathological properties demonstrated that HS injuryinduced neurons to perform a notably disorderly arrangement, loosened cytoplasms and karyopyknosis alterations, and the diffuse vacuolization, interstitial edema was also observed at both 3 and 28 days. The histopathological damage was especially more severe at 3 days than 28 days. Despite the MSC-treated HS rats also showing similar hippocampal histopathological damage, a significant amelioration was observed at both 3 and 28 days when compared with the same time points for untreated HS rats (Figure 4B).

\section{Astrocytes Increased in MSC-Treated HS Rats When Compared With the Untreated Ones}

As shown in Figure 5A, the cytoplasm of the control rats was trachychromatic and closely packed, the intracytoplasmic Nissl bodies were rich. After HS injury, the neurons performed a notably loosened arrangement and diffuse vacuolization, which was characterized by decreased density and an irregular arrangement of the nucleus and Nissl bodies at both 3 and 28 days. Especially, the diffuse vacuolization and decreased Nissl bodies were more notable in the brain of $\mathrm{HS}$ rats at 3 days than at 28 days. Despite the MSC-treated HS rats also showing similar properties of hippocampal neurons in Nissl staining, a significant amelioration was observed at both 3 and 28 days when compared with the same time point for untreated HS rats (Figures 5A,C,D). We further evaluated the astrocyte alterations in the brain of HS rats with or without MSC treatment through immunofluorescence staining of GFAP, which is an astrocyte specific marker. Accompanied by the neuronal alterations, 

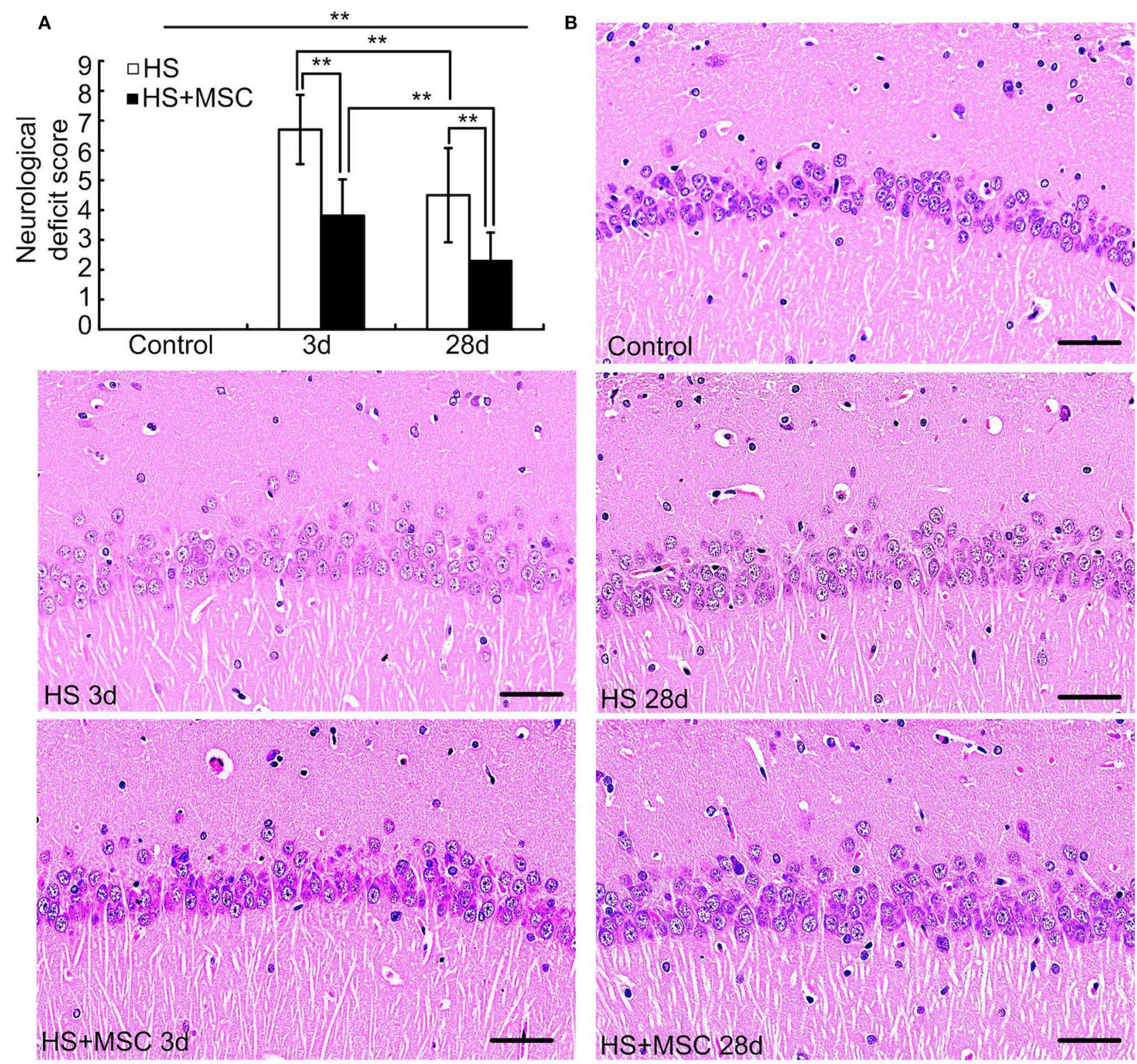

FIGURE 4 | MSC administration provided neuroprotective effects for HS rats. At different time points after MSCs or saline infusion, the neurological deficit scores of rats in each group were tested (A), $n=10$ in each group, and the hippocampal histopathological properties were observed, $n=5$ in each group, the representative images were shown, Scale bar, $50 \mu \mathrm{m}$ (B). ${ }^{\star \star} P<0.01$.

notable decreases in GFAP expression, which indicates astrocytes loss, were observed at both 3 and 28 days after HS injury. MSCtreated HS rats showed approaching normal levels of GFAP expression at both 3 and 28 days when compared with the same time point for untreated HS rats (Figures 5B,E). These results suggested that astrocytes restored in the MSC-treated HS rats when compared with the untreated ones, which indicated the correlation between MSC treatment and astrocytes increment. The further relations and effects between them should be investigated deeply.

\section{MSC Administration Modulates Inflammatory and Chemotactic Cytokines in the Brain Tissue of HS Rats}

Astrocytes are involved in the spatial buffering of many cytokines, signaling molecules, and energy sources that are implicated in the maintenance of homeostasis in the extracellular milieu of neurons. As neuronal damage was mitigated, accompanied with an increment of astrocytes, through MSC administration in HS rats, we further detected the inflammatory and chemotactic cytokines in the brain tissue of HS rats with or without MSC 

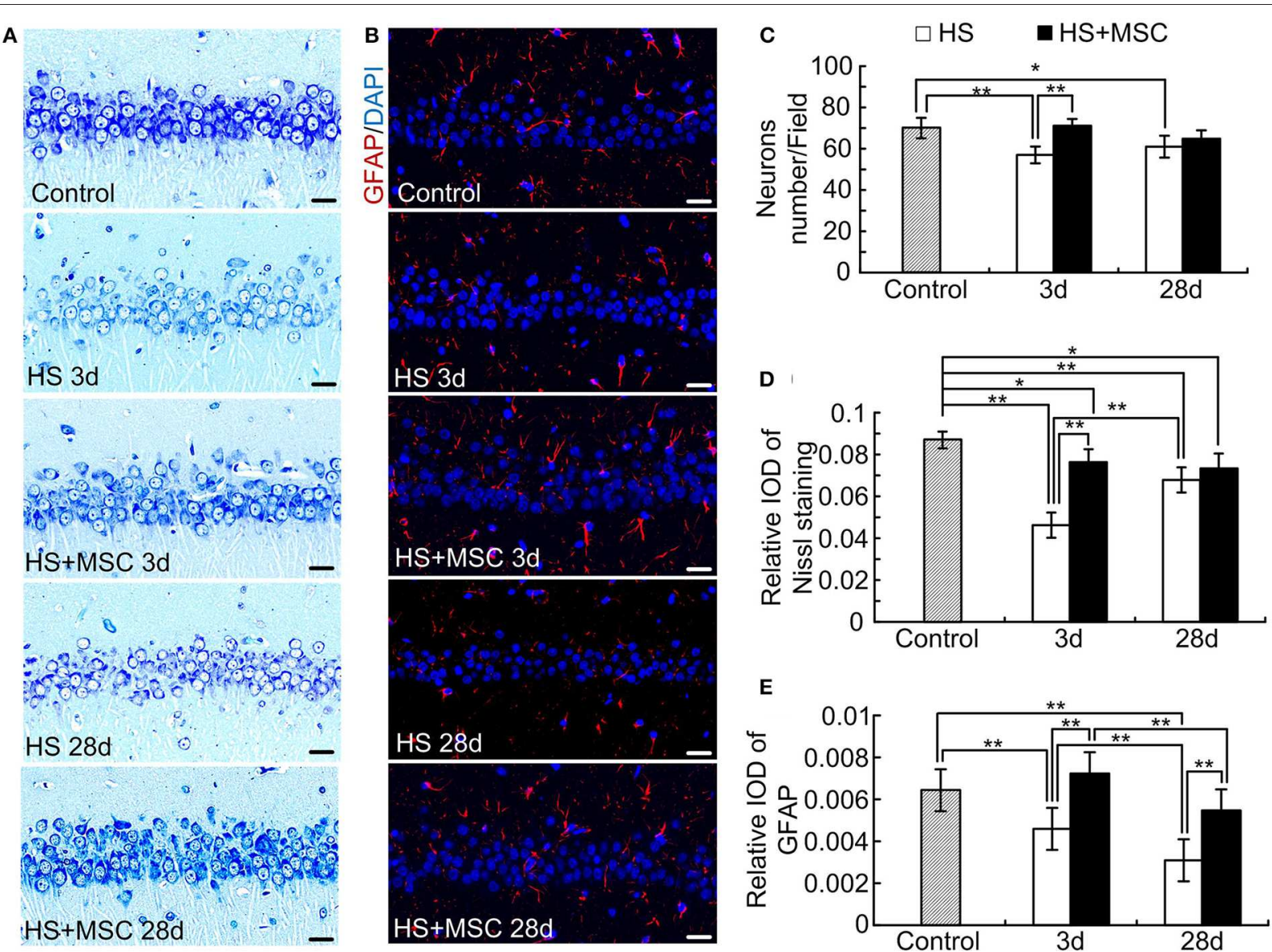

FIGURE 5 | MSCs treatment ameliorated neurons and astrocytes in HS rats. At different time points after MSCs or saline infusion, the Niss staining method in the hippocampus was used to assess the neuron alterations (A), and the immunofluorescence staining of GFAP in the hippocampus was used to assess the astrocytes alterations (B). The representative images were shown, Scale bar, $20 \mu \mathrm{m}$. The neuron count (C) and optical density of Nissl staining (D) and GFAP (E) were analyzed. $n=5$ in each group, ${ }^{\star} P<0.05,{ }^{\star \star} P<0.01$.

administration. As shown in Figure 6, following HS injury the levels of IL-1 $\beta$, IL- 6 , and TNF- $\alpha$ in the rats' brains were significantly increased at 3 days when compared with those of the control rats $(P<0.01)$. Despite the IL-1 $\beta$, IL-6, and TNF- $\alpha$ levels being notably decreased at 28 days when compared with those at 3 days $(P<0.01)$, they were still significantly increased when compared with that of the control group $(P<0.05$ or 0.01$)$. MSC-treated HS rats demonstrated a significant improvement in the levels of brain inflammatory cytokines. Compared with untreated rats, the levels of IL- $1 \beta$ and TNF- $\alpha$ in the brain tissue of MSC-treated rats were both significantly decreased at 3 days $(P<0.01)$, but had no differences at 28 days (Figures 6A,C). IL-6 levels of the MSC-treated rats were significantly decreased when compared with those of untreated rats at both 3 and 28 days (Figure 6B, $P<0.01$ ). This lasted until day 28, when the abovementioned cytokines all approached normal levels in the brains of MSC-treated HS rats (Figures 6A-C).

After HS injury, the rat's brain GRO, MCP-1, and Rantes levels were significantly increased at 3 and 28 days when compared with those of the control rats $(P<0.05$ or 0.01$)$. At 28 days, the GRO and MCP-1 both significantly decreased $(P<0.01)$, but Rantes had no differences when compared with rats of the 3-day group (Figures 6D-F). Compared with untreated rats, the brain GRO and MCP-1 levels of MSC-treated rats were both significantly decreased at 3 days but had no differences at 28 days (Figures 6D,E). The Rantes levels of MSC-treated rats were still significantly decreased when compared with those of the untreated rats at 28 days (Figure 6F). However, brain tissue GRO and MCP-1 levels were still notably higher, and Rantes levels had no differences when compared with those of the control group (Figures 6D-F).

\section{DISCUSSION}

Heat stroke (HS) is the most serious form of heat illness and is characterized by systemic organ damage and central nervous system (CNS) dysfunction $(1,2)$. The morbidity and mortality of $\mathrm{HS}$ are both increased yearly $(3,4)$. Unfortunately, no effective and comprehensive management for HS-induced organ dysfunction, especially for CNS dysfunction, exists. Our present 

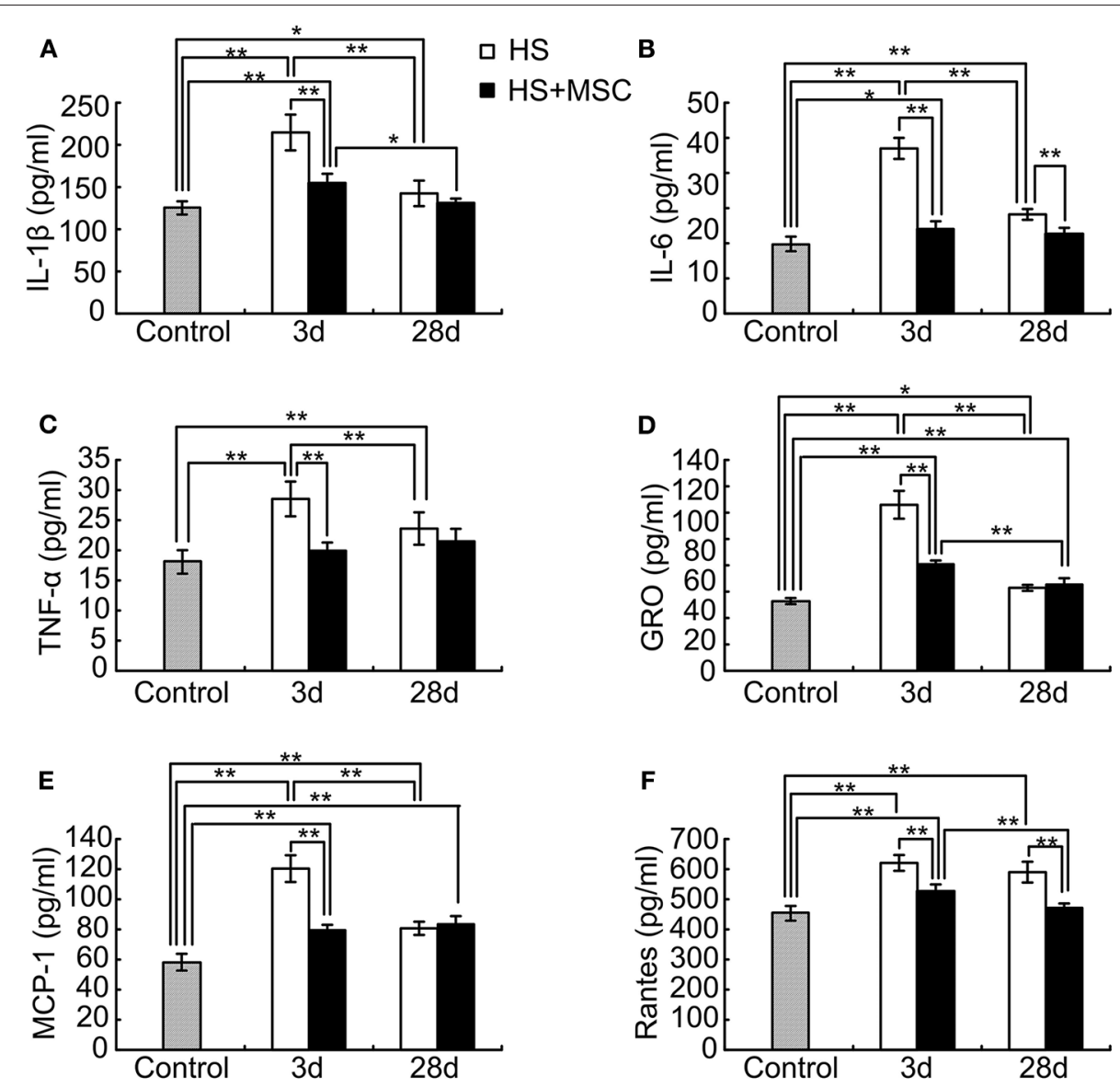

FIGURE 6 | MSC administration modulates inflammatory and chemotactic cytokines in the brain tissue of HS rats. At different time points after MSCs or saline infusion, the brain tissue of rats in each group were collected and homogenized in 10 volumes of ice-cold PBS. The IL-1 $\beta$ (A), IL-6 (B), TNF- $\alpha$ (C), IL-10 (D), MCP-1 (E), and Rantes $\mathbf{( F )}$ levels in the rat's brain tissue lysates were assayed. $n=10$ rats per group. ${ }^{\star} P<0.05,{ }^{\star \star} P<0.01$.

study demonstrated that adipose-derived mesenchymal stem cell (MSCs) administration is a potential treatment strategy for HSinduced system inflammation and CNS injury in rats. Moreover, astrocytes increased in MSC-treated HS rats when compared with the untreated ones.

According to clinical data, the mortality of HS is over $60 \%$ $(3,4)$. Our present study indicated that HS rats were categorized by a high colonic temperature $\left(42^{\circ} \mathrm{C}\right)$ and validated with acute hindlimb paralysis. Moreover, untreated HS rats exhibited a high mortality both in the early (3 days) and long-lasting stages (28 days). Following an infusion of MSCs, the colonic temperature of the rats decreased quickly; the survival rate demonstrated a significant amelioration both in the early (3 days) and long-lasting stages (28 days). These results indicate that HS injury induced a paralysis symptom and high mortality in rats, which could be ameliorated by MSC infusion. Weise et al. (23) reported a negative result when applying cryopreserved HUCB mononuclear cells into the treatment of stroke rats, which indicated potent problems in using cryopreserved cells. Actually, cryopreserved MSCs can be utilized in experiments in two ways. The cryopreserved cells can be resuscitated and then directly transfused into veins. Or the cells should be resuscitated and then recultured for several generations before intravenous infusion. The former way as Weise et al. (23) reported, might be prone to lead to negative results. In the present study, the latter way was selected, and the positive results suggested its effectiveness. Following the HS injury models induction, the decrease of the rats' temperature was related to inflammation modulation and physical heat dissipation. The phenomenon that the temperature of the treated group dropped more quickly than that of the HS group was observed, while the mechanism of it is still unclear and just based on conjecture. The infused MSC's prompt effect of modulating the system inflammatory reaction may contribute to the temperature decrease. But the cells should not have direct affection on the heat dissipation as they were very few in number and volume. The deeper mechanism on how infused MSC can cool down the HS rats so quickly is to be studied further. A previous study has also demonstrated that the survival rate of rats in the interstitial stem cell intervention group was significantly higher than in the HS group, and the earlier the mesenchymal stem cells were injected, the higher the survival rate (18). In addition, the survival 
rate, severity, and prognosis of HS were mainly associated with the intense system inflammatory reaction, increased vascular permeability, abnormal coagulation, cell dysfunction, and finally multi-organ failure (24-26). Therefore, MSC-mediated systemic inflammatory reactions in HS rats should be explored.

The results of the current study indicated that MSC infusion significantly restores serum IL-10 levels and inhibits the elevation of IL-1 $\beta$, IL- 6 , and TNF- $\alpha$ levels at the early stage phase (3 days). At 28 days, the IL- 6 levels were still significantly inhibited by the MSC infusion, but IL-10, IL-6, and TNF- $\alpha$ levels showed no significant differences between the MSCs-treated and untreated rats. High temperatures induced direct cell damage, secondary damage to the intestine and other organs, a coagulation disorder, and systemic inflammatory response syndrome which made the pathological process similar to sepsis $(1,6,27)$. Sepsis is a potentially lethal syndrome that can induce both proinflammatory and anti-inflammatory mechanisms to become a disorder (28). In addition, previous studies have demonstrated that the cytokine-mediated systemic inflammatory response plays an important role in HS development $(1,6,27)$. IL- $1 \beta$, IL-6, and TNF- $\alpha$ are considered pro-inflammatory cytokines which can induce acute inflammatory reactions. Moreover, IL6 and TNF- $\alpha$ levels were notably correlated with the severity and mortality in different animal HS models $(29,30)$. IL-10 is a key anti-inflammatory cytokine produced by activated immune cells, previous studies have demonstrated that treatment with CD34+ cells causes a significant increase in the serum levels of IL-10 during heatstroke (31). In addition, administration of human umbilical cord blood cells (HUCBC) also increases the serum levels of IL-10 and decreases the levels of TNF- $\alpha$ during heat stroke (19). These results demonstrate that the modulation of inflammatory cytokines in the serum may be an important role of MSCs against HS injury. MSCs possess multipotent properties, such as the capacity to secrete various factors, differentiation potential, and immunoregulatory effects (14). Many previous studies have confirmed that MSCs can perform notably antiinflammatory effects, improving organ function, and ultimately reducing the mortality of sepsis (32-34). Our present study and other results raise the possibility that MSCs may be beneficial for protecting against multi-organ dysfunction during heat stroke.

CNS dysfunction or injury is a notable characteristic of HS. Our previous research has demonstrated that $100 \%$ of HS patients have CNS dysfunction, and the incidence of long-term neurological dysfunction reached $24.4 \%$ (35). Other research has also reported that all HS patients have CNS injury, while more than $30 \%$ of them will progress to long-term CNS dysfunction as sequela $(3,5)$. Our present study demonstrated that the neurological deficit and hippocampal histopathological properties were significantly ameliorated in MSC-treated HS rats at both 3 and 28 days when compared with the same time points for untreated HS rats. The neurological deficits were evaluated by a modified Neurological Severity Score system which is primarily used in ischemic stroke. However, Boltze et al. (36) reported that the system was not very sensitive and could be prone to compensation, which indicated the probable inaccuracy of the scores. Our present study showed that there were significant differences in scores between either
$3 \mathrm{~d}$ groups or $28 \mathrm{~d}$ groups. The comparison between HS group and MSC group at the same time points (i.e., untreated $3 \mathrm{~d}$ group vs. MSC-treated 3d group, untreated 28d group vs. MSC-treated 28d group) might offset partial compensation. Nevertheless, other behavior tests (such as the cylinder task, tapered beam walking, foot fault and Montoya's staircase) which are minimally affected by subjective scoring, compensation or repeated measurements should be considered to be applied to evaluate neurological deficits in further studies (37). The hippocampus of the brain is particularly vulnerable to various injury factors which would induce permanent neurological sequelae. During HS, heat damage can induce hypotension and intracranial hypertension which reduce blood flow to the brain and result in cerebral ischemia, hypoxia, and neuronal damage $(19,38)$. A secondary neurotoxic cascade which contains the reactive oxygen species (ROS), cytokines, glutamate, and calcium ion overload further exacerbates the neuronal damage $(19,38)$. Another researcher reported that HS can destroy learning ability and memory in rats, the mechanisms of which may be associated with iron level alterations in the hippocampus (9). Despite the pathogenesis being clear, rapid and effective treatments are still deficient. Currently, MSC-based therapy is being explored as a potential treatment strategy for CNS injury. MSCs can play potential neuronal protective effects in strokes, traumatic brain injuries, multiple sclerosis, nerve injuries, and neurodegenerative diseases (11-13). The specific mechanisms contain the secretion of neurotrophic factors, regulation of inflammatory reactions, modulation of ion metabolism, and so on (11-13). Therefore, the present results indicate that the neuroprotective effects by MSC infusion on HS rats may be associated with these MSC properties. In addition, we also demonstrated that MSC-treated HS rats exhibited a notable amelioration of hippocampal neurons, which was accompanied with restored astrocytes.

Astrocytes are involved in the spatial buffering of many ions, signaling molecules, and energy sources that are implicated in maintaining the homeostasis of the extracellular milieu of neurons. A previous study reported that murine astrocytes regulated chemokine expression and triggered cell survival ex vivo after HS (39). Another study found that MSC treatment restored astrocytic endfeet in the inflammatory brain (40). In our study, the increment of astrocytes in MSC-treated rats was founded. However, on the basis of present data, the mechanism of how MSCs reached the effects is still unclear. Besides, to validate whether astrocytes are responsible for the therapeutic effects, an astrocyte subtype analysis and more detailed investigation are required (41). Similar to other diseases, such as stroke, neuro-inflammation is the main contributor to CNS dysfunction in the HS condition. In the process of heat stress, proinflammatory factors and chemokines increase quickly, and a long-term increase in CNS cytokines and chemokines contribute to the consequences of CNS damage during heat stroke (42, 43). Our present study has demonstrated that MSC-treated HS rats demonstrated a significant improvement in the levels of brain inflammatory cytokines. Moreover, the brain GRO, MCP-1, and Rantes levels of MSC-treated rats were both significantly decreased. All of these results demonstrate that excessive activation of inflammation in the brain may be a 
major pathological mechanism of HS. Thereby, modulation of cerebral inflammatory response may be considered a potential management strategy for HS prevention and therapy.

In summary, the results of the present study indicate that MSC treatment may ameliorate the survival rate of rats by regulating circulation and cerebral inflammatory response. Moreover, astrocytes increased in MSC-treated HS rats when compared with the untreated ones. These may suggest a potential mechanism for HS prevention and therapy by MSC administration.

\section{DATA AVAILABILITY STATEMENT}

The datasets generated for this study are available on request to the corresponding author.

\section{ETHICS STATEMENT}

The animal study was reviewed and approved by Animal Care and Use Committee of the General Hospital of PLA.

\section{AUTHOR CONTRIBUTIONS}

YuZ helped to design and perform the experiments and wrote the draft of the manuscript. ZD helped to design the

\section{REFERENCES}

1. Bouchama A, Knochel JP. Heat stroke. N Engl J Med. (2002) 346:197888. doi: 10.1056/NEJMra011089

2. Leon LR, Bouchama A. Heat stroke. Compr Physiol. (2015) 5:61147. doi: 10.1002/cphy.c140017

3. Argaud L, Ferry T, Le QH, Marfisi A, Ciorba D, Achache P, et al. Short- and long-term outcomes of heatstroke following the 2003 heat wave in Lyon, France. Arch Intern Med. (2007) 167:2177-83. doi: 10.1001/archinte.167.20.ioi70147

4. Hausfater P, Megarbane B, Dautheville S, Patzak A, Andronikof M, Santin $\mathrm{A}$, et al. Prognostic factors in non-exertional heatstroke. Intensive Care Med. (2010) 36:272-80. doi: 10.1007/s00134-009-1694-y

5. Sharma HS, Hoopes PJ. Hyperthermia induced pathophysiology of the central nervous system. Int J Hyperthermia. (2003) 19:325-54. doi: 10.1080/0265673021000054621

6. Huisse MG, Pease S, Hurtado-Nedelec M, Arnaud B, Malaquin C, Wolff M, et al. Leukocyte activation: the link between inflammation and coagulation during heatstroke. A study of patients during the 2003 heat wave in Paris. Crit Care Med. (2008) 36:2288-95. doi: 10.1097/CCM.0b013e318180dd43

7. Trujillo MH, Bellorin-Font E, Fragachan CF, Perret-Gentil R. Multiple organ failure following near fatal exertional heat stroke. J Intensive Care Med. (2009) 24:72-8. doi: 10.1177/0885066608327122

8. Hsu SF, Niu KC, Lin CL, Lin MT. Brain cooling causes attenuation of cerebral oxidative stress, systemic inflammation, activated coagulation, and tissue ischemia/injury during heatstroke. Shock. (2006) 26:21020. doi: 10.1097/01.shk.0000223124.49265.10

9. Liu J, Wan M, Zhang Y, Zhang S, Zhang H, Wu S. Dysfunction of iron metabolism and iron-regulatory proteins in the rat hippocampus after heat stroke. Shock. (2019) 51:780-6. doi: 10.1097/SHK.0000000000001182

10. Hamaya H, Hifumi T, Kawakita K, Okazaki T, Kiridume K, Shinohara $\mathrm{N}$, et al. Successful management of heat stroke associated with multipleorgan dysfunction by active intravascular cooling. Am J Emerg Med. (2015) 33:124.e5-7. doi: 10.1016/j.ajem.2014.05.056

11. Alia C, Terrigno M, Busti I, Cremisi F, Caleo M. Pluripotent stem cells for brain repair: protocols and preclinical applications study, perform the experiments, and analyze the results. YL helped to perform the experiments, analyze the results, and revise the manuscript. RY helped to perform the experiments. MY helped to analyze the results. YaZ helped to revise the manuscript. LW helped to perform the experiments. FZ helped to develop the study rationale and design. HK helped to conceive, design, and supervise the study and analyzed the results.

\section{FUNDING}

This work was supported by grants of The National Natural Science Foundation of China [No. 81671966]; the Cultivation Program for Military Medical Science and Technology Youth-Growth Project [No. 16QNP139]; and Application Research and Achievement Extension of Clinical Characteristics in Chinese Capital Foundation [No. Z171100001017160].

\section{SUPPLEMENTARY MATERIAL}

The Supplementary Material for this article can be found online at: https://www.frontiersin.org/articles/10.3389/fneur. 2020.00372/full\#supplementary-material in cortical and hippocampal pathologies. Front Neurosci. (2019) 13:684. doi: $10.3389 /$ fnins.2019.00684

12. Hsuan YC, Lin CH, Chang CP, Lin MT. Mesenchymal stem cell-based treatments for stroke, neural trauma, and heat stroke. Brain Behav. (2016) 6:e00526. doi: 10.1002/brb3.526

13. Kalladka D, Muir KW. Brain repair: cell therapy in stroke. Stem Cells Cloning. (2014) 7:31-44. doi: 10.2147/SCCAA.S38003

14. Orbay H, Tobita M, Mizuno H. Mesenchymal stem cells isolated from adipose and other tissues: basic biological properties and clinical applications. Stem Cells Int. (2012) 2012:461718. doi: 10.1155/2012/461718

15. Kong D, Zhu J, Liu Q, Jiang Y, Xu L, Luo N, et al. Mesenchymal stem cells protect neurons against hypoxic-ischemic injury via inhibiting parthanatos, necroptosis, and apoptosis, but not autophagy. Cell Mol Neurobiol. (2017) 37:303-13. doi: 10.1007/s10571-016-0370-3

16. Cheng Q, Zhang Z, Zhang S, Yang H, Zhang X, Pan J, et al. Human umbilical cord mesenchymal stem cells protect against ischemic brain injury in mouse by regulating peripheral immunoinflammation. Brain Res. (2015) 1594:293304. doi: 10.1016/j.brainres.2014.10.065

17. Lv W, Li WY, Xu XY, Jiang H, Bang OY. Bone marrow mesenchymal stem cells transplantation promotes the release of endogenous erythropoietin after ischemic stroke. Neural Regen Res. (2015) 10:1265-70. doi: 10.4103/1673-5374.162759

18. Lin W, Hsuan YC, Su YC, Lin CH, Lin MT, Chen ZH, et al. CD34(-) human placenta-derived mesenchymal stem cells protect against heat stroke mortality in rats. Oncotarget. (2018) 9:1992-2001. doi: 10.18632/oncotarget. 23324

19. Tseng LS, Chen SH, Lin MT, Lin YC. Umbilical cord blood-derived stem cells improve heat tolerance and hypothalamic damage in heat stressed mice. Biomed Res Int. (2014) 2014:685683. doi: 10.1155/2014/685683

20. Deng Z, Xu H, Zhang J, Yang C, Jin L, Liu J, et al. Infusion of adiposederived mesenchymal stem cells inhibits skeletal muscle mitsugumin 53 elevation and thereby alleviates insulin resistance in type 2 diabetic rats. Mol Med Rep. (2018) 17:8466-74. doi: 10.3892/mmr.2018.8901

21. Hu J, Kang HJ, Liu C, Hu P, Yang MM, Zhou FH. Response of regulatory T cells to classic heat stroke in mice. Exp Ther Med. (2018) 16:460915. doi: $10.3892 / \mathrm{etm} .2018 .6766$ 
22. Chen J, Sanberg PR, Li Y, Wang L, Lu M, Willing AE, et al. Intravenous administration of human umbilical cord blood reduces behavioral deficits after stroke in rats. Stroke. (2001) 32:2682-8. doi: 10.1161/hs1101.098367

23. Weise G, Lorenz M, Pösel C, Maria Riegelsberger U, Störbeck V, Kamprad $\mathrm{M}$, et al. Transplantation of cryopreserved human umbilical cord blood mononuclear cells does not induce sustained recovery after experimental stroke in spontaneously hypertensive rats. J Cereb Blood Flow Metab. (2014) 34:e1-9. doi: 10.1038/jcbfm.2013.185

24. Hifumi T, Kondo Y, Shimizu K, Miyake Y. Heat stroke. J Intensive Care. (2018) 6:30. doi: 10.1186/s40560-018-0298-4

25. Tao Z, Cheng M, Wang SC, Lv W, Hu HQ, Li CF, et al. JAK2/STAT3 pathway mediating inflammatory responses in heatstroke-induced rats. Int J Clin Exp Pathol. (2015) 8:6732-9.

26. Yan YE, Zhao YQ, Wang H, Fan M. Pathophysiological factors underlying heatstroke. Med Hypotheses. (2006) 67:60917. doi: 10.1016/j.mehy.2005.12.048

27. Leon LR, Helwig BG. Heat stroke: role of the systemic inflammatory response. J Appl Physiol. (2010) 109:1980-8. doi: 10.1152/japplphysiol.003 01.2010

28. Epstein Y, Roberts WO, Golan R, Heled Y, Sorkine P, Halpern P. Sepsis, septic shock, and fatal exertional heat stroke. Curr Sports Med Rep. (2015) 14:64-9. doi: 10.1249/JSR.0000000000000112

29. Bouchama A, Roberts G, Al Mohanna F, El-Sayed R, Lach B, CholletMartin S, et al. Inflammatory, hemostatic, and clinical changes in a baboon experimental model for heatstroke. J Appl Physiol. (2005) 98:697705. doi: 10.1152/japplphysiol.00461.2004

30. Lu KC, Wang JY, Lin SH, Chu P, Lin YF. Role of circulating cytokines and chemokines in exertional heatstroke. Crit Care Med. (2004) 32:399403. doi: 10.1097/01.CCM.0000108884.74110.D9

31. Chen SH, Chang FM, Chang HK, Chen WC, Huang KF, Lin MT. Human umbilical cord blood-derived CD34+ cells cause attenuation of multiorgan dysfunction during experimental heatstroke. Shock. (2007) 27:663-71. doi: 10.1097/01.shk.0000248593.71388.40

32. Chao YH, Wu HP, Wu KH, Tsai YG, Peng CT, Lin KC, et al. An increase in $\mathrm{CD} 3+\mathrm{CD} 4+\mathrm{CD} 25+$ regulatory $\mathrm{T}$ cells after administration of umbilical cord-derived mesenchymal stem cells during sepsis. PLoS ONE. (2014) 9:e110338. doi: 10.1371/journal.pone.0110338

33. Johnson CL, Soeder Y, Dahlke MH. Concise Review: mesenchymal stromal cell-based approaches for the treatment of acute respiratory distress and sepsis syndromes. Stem Cells Transl Med. (2017) 6:114151. doi: $10.1002 /$ sctm.16-0415

34. Sung PH, Chiang HJ, Chen $\mathrm{CH}$, Chen YL, Huang TH, Zhen YY, et al. Combined therapy with adipose-derived mesenchymal stem cells and ciprofloxacin against acute urogenital organ damage in rat sepsis syndrome induced by intrapelvic injection of cecal bacteria. Stem Cells Transl Med. (2016) 5:782-92. doi: 10.5966/sctm.2015-0116

35. Yang M, Li Z, Zhao Y, Zhou F, Zhang Y, Gao J, et al. Outcome and risk factors associated with extent of central nervous system injury due to exertional heat stroke. Medicine. (2017) 96:e8417. doi: 10.1097/MD.0000000000008417

36. Boltze J, Lukomska B, Jolkkonen J. Mesenchymal stromal cells in stroke: improvement of motor recovery or functional compensation? J Cereb Blood Flow Metab. (2014) 34:1420-1. doi: 10.1038/jcbfm.2014.94

37. Balkaya MG, Trueman RC, Boltze J, Corbett D, Jolkkonen J. Behavioral outcome measures to improve experimental stroke research. Behav Brain Res. (2018) 352:161-71. doi: 10.1016/j.bbr.2017.07.039

38. Chen SH, Lin MT, Chang CP. Ischemic and oxidative damage to the hypothalamus may be responsible for heat stroke. Curr Neuropharmacol. (2013) 11:129-40. doi: 10.2174/1570159X11311020001

39. Choi K, Ni L, Jonakait GM. Fas ligation and tumor necrosis factor $\alpha$ activation of murine astrocytes promote heat shock factor-1 activation and heat shock protein expression leading to chemokine induction and cell survival. $J$ Neurochem. (2011) 116:438-48. doi: 10.1111/j.1471-4159.2010.07124.x

40. Park HJ, Shin JY, Kim HN, Oh SH, Song SK, Lee PH. Mesenchymal stem cells stabilize the blood-brain barrier through regulation of astrocytes. Stem Cell Res Ther. (2015) 6:187. doi: 10.1186/s13287-015-0180-4

41. Wagner DC, Scheibe J, Glocke I, Weise G, Deten A, Boltze J, et al. Objectbased analysis of astroglial reaction and astrocyte subtype morphology after ischemic brain injury. Acta Neurobiol Exp. (2013) 73:79-87.

42. Lee W, Moon M, Kim HG, Lee TH, Oh MS. Heat stress-induced memory impairment is associated with neuroinflammation in mice. $J$ Neuroinflammation. (2015) 12:102. doi: 10.1186/s12974-015-0324-6

43. Lin YF, Liu TT, Hu CH, Chen CC, Wang JY. Expressions of chemokines and their receptors in the brain after heat stroke-induced cortical damage. $J$ Neuroimmunol. (2018) 318:15-20. doi: 10.1016/j.jneuroim.2018.01.014

Conflict of Interest: The authors declare that the research was conducted in the absence of any commercial or financial relationships that could be construed as a potential conflict of interest.

Copyright (c) 2020 Zhang, Deng, Li, Yuan, Yang, Zhao, Wang, Zhou and Kang. This is an open-access article distributed under the terms of the Creative Commons Attribution License (CC BY). The use, distribution or reproduction in other forums is permitted, provided the original author(s) and the copyright owner(s) are credited and that the original publication in this journal is cited, in accordance with accepted academic practice. No use, distribution or reproduction is permitted which does not comply with these terms. 